\title{
Evolution of arsenic in high fluence plasma immersion ion implanted silicon: behavior of the as-implanted surface
}

\author{
V. Vishwanath ${ }^{1}$, E. Demenev ${ }^{2,3}$, D. Giubertoni ${ }^{2, a}$, L. Vanzetti ${ }^{2}$, A. L. Koh ${ }^{4}$,

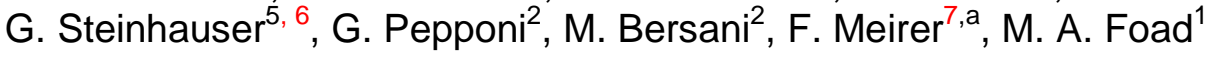 \\ ${ }^{1}$ Applied Materials, 3225 Oakmead Village Drive, Santa Clara, CA 95052, USA \\ ${ }^{2}$ Center for Materials and Microsystems, Fondazione Bruno Kessler, Via Sommarive 18, 38123 Povo, \\ Trento, Italy \\ ${ }^{3}$ Dipartment of Molecular Science and Nanosystems, Ca'Foscari University, Dorsoduro 2137, 30123, \\ Venice, Italy \\ ${ }^{4}$ Stanford Nanocharacterization Laboratory, Stanford University, 476 Lomita Mall, Stanford, CA 94305 \\ USA \\ ${ }^{5}$ Colorado State University, Environmental and Radiological Health Sciences, Fort Collins, CO 80523 \\ USA \\ ${ }^{6}$ Leibniz Universität Hannover, Institut für Radioökologie und Strahlenschutz, 30419 Hannover, Germany \\ ${ }^{7}$ Inorganic Chemistry and Catalysis, Utrecht University, Utrecht 3584 CG, Netherlands
}

High fluence $\left(>10^{15}\right.$ ions $\left./ \mathrm{cm}^{2}\right)$ low-energy $\left(<2 \mathrm{keV}\right.$ ) plasma immersion ion implantation (PIII) of $\mathrm{AsH}_{3}{ }^{+}$ on (100) silicon was investigated, with the focus on stability and retention of the dopant. At this dose, a thin $(\sim 3 \mathrm{~nm})$ amorphous layer forms at the surface, which contains about $45 \%$ arsenic (As) in a silicon and oxygen matrix. The presence of silicon indicates that the layer is not only a result of deposition, but predominantly ion mixing. High fluence PIII introduces high concentration of arsenic, modifying the stopping power for incoming ions resulting in an increased deposition. When exposed to atmosphere, the arsenic rich layer spontaneously evolves forming arsenolite $\mathrm{As}_{2} \mathrm{O}_{3}$ micro-crystals at the surface. The micro-crystal formation was monitored over several months and exhibits typical crystal growth kinetics. At the same time, a continuous growth of native silicon oxide rich in arsenic was observed on the exposed surface, suggesting the presence of oxidation enhancing factors linked to the high arsenic concentration at the surface.

a) corresponding authors: giuberto@fbk.eu, f.meirer@uu.nl

\section{INTRODUCTION}

The evolution of a microprocessor with its increasing complexity in the transistor geometry calls for advanced doping solutions, particularly to address electrical characteristics of transistors in a three dimensional structure. Traditional beamline ion implantation produces inhomogeneous doping concentrations on the top and the side walls of a three dimensional transistor. In particular, shielding and elastic scattering from the walls 
prevent achieving the desired level of dopant retention [1,2]. Plasma immersion ion implantation (PIII) can be suitable for those applications since it can ensure a more conformal doping coupled with the possibility of achieving ultra-shallow profiles with higher fluences and high throughput for high volume production [3]. However, PIII process integration is not straightforward since the exposure of Si wafers to plasma can also result in etching [4], deposition, and implantation, depending on the plasma conditions. Recently reported results shows a relatively thick $(5-10 \mathrm{~nm})$ native oxide rich in arsenic after high fluence PIII on Si surfaces [2,5-8].

Meirer et al. recently demonstrated that the thick native oxide observed after high fluence PIII of arsenic in $\mathrm{Si}$ originates from atmospheric exposure of the implanted surface [9]. In fact, in-situ deposition of a cap layer on the implanted surface prevents further oxidation. The same authors also reported that exposing the implanted surface to atmosphere results in the formation of arsenolite micro crystals (cubic $\mathrm{As}_{2} \mathrm{O}_{3}$, space group $\mathrm{Fd} 3 \mathrm{~m}$ ). This formation of micro crystals resulted in an arsenic dose loss upon annealing, since $\mathrm{As}_{2} \mathrm{O}_{3}$ easily sublimates at temperatures as low as $200^{\circ} \mathrm{C}$ without any appreciable indiffusion of arsenic. More recently, Meirer et al. reported that in the first few months of atmospheric exposure, a second mechanism of arsenic loss beside the micro crystal formation is observed while the surface oxide continues to grow [10]. As a possible explanation for those observations the authors suggested that a thin film of amorphous arsenic was deposited following the high fluence PIII. This arsenic layer would then evolve upon atmospheric exposure forming $\mathrm{As}_{2} \mathrm{O}_{3}$ microcrystals while the high arsenic concentration at the As/Si interface would cause an enhanced oxidation of the Si surface. On the other hand, if annealing is performed before those reactions take place, most of the As can be driven into the $\mathrm{Si}$, explaining in this way the higher retained arsenic fluences often observed on annealed samples [5,6,9]. However, the presence of this amorphous arsenic layer was only proposed based on the high As concentration estimated by SIMS. In this work, an amorphous layer is directly observed and identified by scanning transmission electron microscopy (STEM) and Raman spectroscopy. Quantitative STEM, x-ray photoelectron spectroscopy (XPS) and extended $x$-ray absorption fine structure spectroscopy (EXAFS) reveal that the composition of this layer is a matrix of arsenic, silicon and oxygen. The evolution of this surface layer, when exposed to atmosphere, was then monitored by several techniques and is described and discussed in this paper.

\section{EXPERIMENTAL}

Two 300 mm (100) Si wafers were irradiated by PIII using an $\mathrm{AsH}_{3} / \mathrm{H}_{2}$ gas mixture, at < $2 \mathrm{kV}$ bias with sub $30 \mathrm{mTorr}$ pressure. PIII conditions were set to implant a nominal arsenic 
fluence of $\sim 1 \times 10^{15} \mathrm{at} / \mathrm{cm}^{2}$. The wafers (S1 and S2) were implanted in a chamber with the base pressure of $10^{-6}$ Torr. After the arsenic PIII on wafer S2, a $1 \mathrm{~nm}$ thick protective film of silicon nitride $\left(\mathrm{SiN}_{\mathrm{x}}\right)$ was deposited in-situ, by low temperature chemical vapor deposition. Once implanted, the wafers were stored in clean wafer boxes, which were exposed to atmosphere without any particular control of humidity and/ or atmospheric composition. Samples of $\mathrm{S} 2$ were wet-etched, to remove the $\mathrm{SiN}_{\mathrm{x}}$ cap and to expose the original surface in order to monitor its evolution in the laboratory. Given the relevant fraction of oxygen in the cap layer, wet-etching was carried out with $1 \%$ HF solution in water for 10 seconds. Samples were then rinsed in ethanol and blow dried using nitrogen.

TEM analysis was carried out in both scanning and transmission modes, to investigate sample cross-sections and identify the film stacking structure in the capped (S2) and uncapped (S1) samples. TEM specimens were prepared by a low energy focused ion beam and were examined using a FEI 80-300 Titan environmental (scanning) transmission electron microscope (STEM) operated at $300 \mathrm{kV}$. For the uncapped sample (S1), an iridium protective layer was deposited at room temperature, which does not perturb the composition profile of the dopant. The TEM is equipped with a spherical aberration corrector in the image forming (objective) lens and an Oxford Xmax SDD EDS detector. The EDS line scans were performed in the STEM mode, with a step size of $0.5 \mathrm{~nm}$.

The samples were also inspected by an optical profilometer (Zeta-200). The optical microscopy module was used to monitor the evolution of arsenolite micro crystals. Inspection was carried out as early as two days after PIII. In order to identify the phase of arsenolite crystals and presence of amorphous arsenic, Raman spectroscopy in the backscattered geometry was used. Raman spectroscopy was performed using a $532 \mathrm{~nm}$ diode laser with an incident power of $10 \mathrm{~mW}$ over a spot of $2 \mu \mathrm{m}$. Spectra were acquired for $10 \mathrm{~s}$ in the Stokes scattering regime between 50 and $1100 \mathrm{~cm}^{-1}$. The crystals did not undergo any structural change during the Raman measurement.

Arsenic fluence and depth distribution were measured by secondary ion mass spectrometry (SIMS) and instrumental neutron activation analysis (INAA). SIMS was performed utilizing a Cameca Wf magnetic sector instrument, with a $300 \mathrm{eV} \mathrm{Cs}^{+}$sputtering beam ( $\sim 5^{\circ}$ incidence angle) and collecting ${ }^{75} \mathrm{As}^{-},{ }^{30} \mathrm{Si}^{-}$and ${ }^{18} \mathrm{O}^{-}$secondary ions in high mass resolution $(M / \Delta M=2,000)$. Details of the SIMS quantification protocol are reported elsewhere [11]. INAA measurements for total arsenic dose quantification were carried out at the TRIGA Mark II reactor of the Atominstitut, Vienna University of Technology, as previously reported $[12,13]$. Samples were irradiated for $1200 \mathrm{~s}$ (neutron flux density approx. $3 \times 10^{12}$ $\mathrm{cm}^{-2} \mathrm{~s}^{-1}$ ) using the pneumatic sample transfer system. After a cooling time of at least 60 
minutes, the ${ }^{76}$ As $\left(T_{1 / 2}=1.0942\right.$ days; $\left.559 \mathrm{keV}\right)$ activities were determined with a $222 \mathrm{~cm}^{3}$ HPGe semiconductor detector. The measurement times were chosen independently for each sample, until the error due to counting statistics was $<5 \%$ rel. for the ${ }^{76}$ As peak in each spectrum. The total number of arsenic atoms in the piece of wafer analyzed was determined by calibration with a reference sample.

XPS measurements were carried out using a VG-Scienta Esca-200 system with a monochromatized $\mathrm{Al} \mathrm{K}_{\alpha}(1486.6 \mathrm{eV})$ source. An overall energy resolution of $0.4 \mathrm{eV}$ is routinely used. For each sample $\mathrm{O} 1 \mathrm{~s}$, Si $2 p$, As $3 d$ and As $2 p$ core levels were collected. Oxide thickness was calculated from the area of the two peaks in the Si $2 p$ core level corresponding to elemental and oxidized silicon [13].

Table I. Arsenic fluence measured by INAA and SIMS

\begin{tabular}{lccc}
\hline Sample & Cap $^{\mathrm{b}}$ & As fluence, INAA $\left(\mathrm{cm}^{-2}\right)$ & As fluence, SIMS $\left(\mathrm{cm}^{-2}\right)$ \\
\hline \hline S1 & - & $(5.2 \pm 0.2) \times 10^{15}$ & - \\
S1-cleaned & a & $(2.5 \pm 0.1) \times 10^{15}$ & $(2.6 \pm 0.2) \times 10^{15}$ \\
S2 & Yes & $(5.7 \pm 0.2) \times 10^{15}$ & $(6.0 \pm 0.5) \times 10^{15}$ \\
S2-etched & Yes & $(3.6 \pm 0.2) \times 10^{15}$ & $(2.9 \pm 0.3) \times 10^{15}$ \\
\hline \multicolumn{4}{l}{${ }^{\text {a }}$ Sample surface was cleaned from the micro-crystals } \\
b Protective cap had been removed by a dilute HF wet etch (1\% in water, $10 \mathrm{~s})$.
\end{tabular}

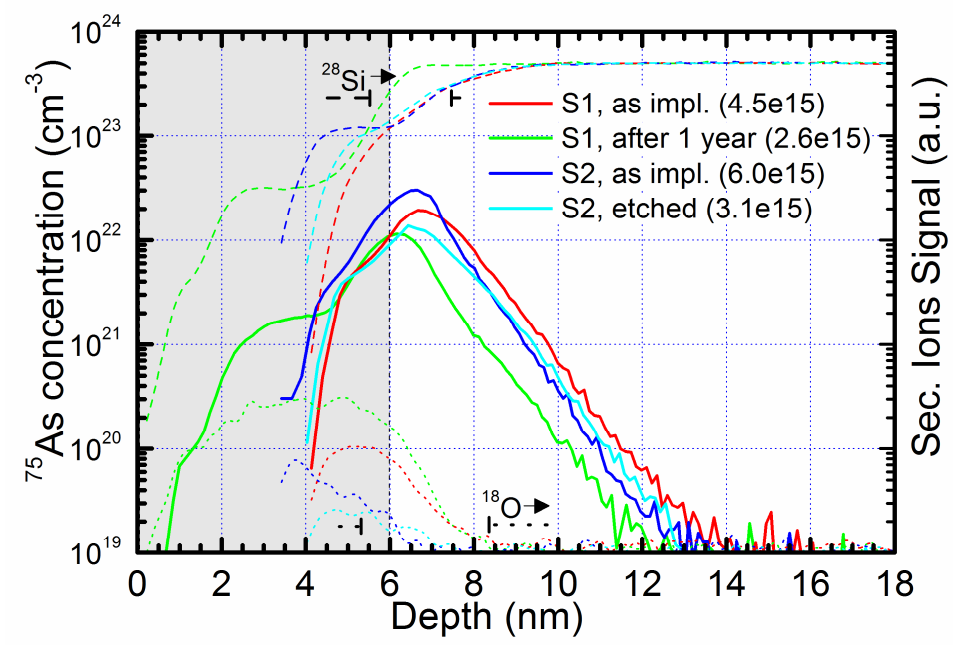

Figure 1. Arsenic SIMS profiles of S1 sample measured after 28 days ("as implanted") and 1 year of atmospheric exposure since PIII, and of S2 sample (28 day old) measured with or without $\mathrm{SiN}_{\mathrm{x}}$ cap (after wet etch). Matrix related species $28 \mathrm{Si}^{-}$(dash) and 180' (dotted) refer to the right axis helping the stack structure identification. The zero of the "Depth" axis was set at the interface between Si and $\mathrm{SiO}_{2}$ or $\mathrm{SiN}_{\mathrm{x}}$ cap for $\mathrm{Si} 1$ and $\mathrm{S} 2$, respectively. 
Grazing incidence x-ray absorption fine structure measurements (GI-XAFS) have been performed in fluorescence mode at the Stanford Synchrotron Radiation Lightsource (SSRL) beamline 4-1, to investigate the local order around arsenic atoms and thus identify the phase of deposited/implanted arsenic. The angle of incidence was set to $0.34^{\circ}$, which is well above the critical angle of external total reflection of x-rays of the selected energy and a $\mathrm{Si}$ substrate at $\sim 0.15^{\circ}$. This angle was selected on the basis of the $x$-ray penetration depth into the substrate to ensure that the entire arsenic distribution was probed [15-17]. For the sample $\mathrm{S} 1$ an additional $\mathrm{Gl}$ angle ( 0.059 was selec ted to restrict the probing depth to the top $3 \mathrm{~nm}$ and to exclusively examine the near surface layer and the expected arsenolite crystals on the surface of the Si wafer.

\section{RESULTS}

\subsection{SURFACE LAYER COMPOSITION}

In order to evaluate the effectiveness of the $\mathrm{SiN}_{\mathrm{x}}$ cap in preserving the original PIII irradiated surface, both INAA and SIMS analysis were carried out to measure arsenic fluence and depth distribution. Fluence values are summarized in Table I and overall a good agreement between the two analytical techniques is obtained. The fluence measured on sample $\mathrm{S} 2$ is the highest for both SIMS and INAA, i.e. $\sim 6 \times 10^{15}$ at $/ \mathrm{cm}^{2}$ suggesting that the $\mathrm{SiN}_{\mathrm{x}}$ cap helps in retaining most of the implanted arsenic. The fluence measured on sample S1 by INAA is $10 \%$ lower than that for S2. However, INAA S1 fluence was twice the one measured for S1cleaned by SIMS, $5.2 \times 10^{15} \mathrm{at} / \mathrm{cm}^{2}$ against $2.6 \times 10^{15}$ at $/ \mathrm{cm}^{2}$, respectively. Here it is important to note that SIMS measures only the arsenic within the wafer, since micro crystals were removed before analysis due to a routine surface cleaning prior to the measurement. Therefore, the difference in fluence was attributed to the presence of arsenolite micro crystals [9]. When micro-crystals are gently brushed off from the wafer surface, the dose determined by INAA decreases from $5.2 \times 10^{15}$ (S1) to $2.5 \times 10^{15} \mathrm{at} / \mathrm{cm}^{2}$ (S1-cleaned), and are then in agreement with SIMS (within experimental uncertainty). Therefore, by comparing the INAA S1 fluence with the SIMS data, it can be concluded that at least $(2.6 \pm 0.3) \times 10^{15} \mathrm{at} / \mathrm{cm}^{2}$ of the As measured on $\mathrm{S} 1$ was involved in the arsenolite phase. The $\mathrm{SiN}_{\mathrm{x}}$ cap of $\mathrm{S} 2$ prevented this arsenolite formation and any relevant loss of As. SIMS depth profiles are reported in Figure 1, which confirms the effectiveness of the cap in preserving the original irradiated surface. Furthermore, the S2 profile is stable during atmospheric exposure, whereas S1 depth distributions evolve, not only with the micro crystal formation but also, with a continuous growth of the silicon oxide, re-distribution of arsenic in the silicon oxide and the decrease in the total fluence [10]. 
Amorphous

Epoxy

arsenic rich

layer

$\operatorname{SiN}_{x}$

Silicon

substrate

[20 nm

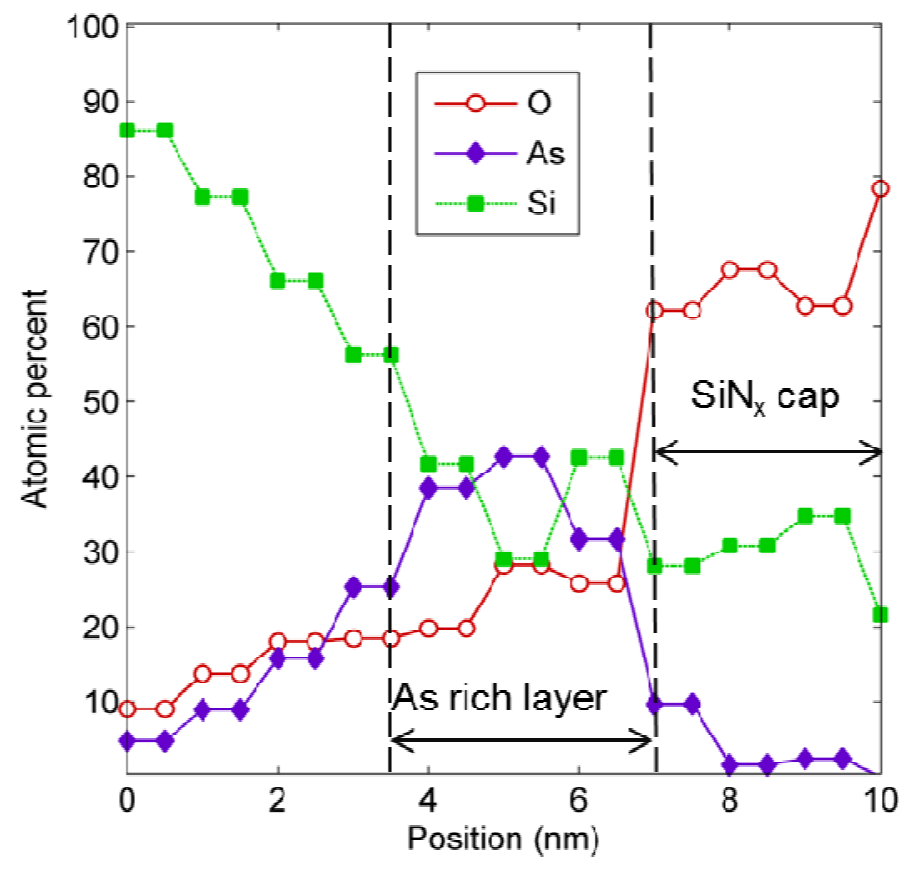

Figure 2. A dark field cross-section STEM image (top) and an EDS line scan (bottom) on S2 (capped sample). The amorphous arsenic rich layer which has been retained by the cap, can be seen as a $\sim 3$ $\mathrm{nm}$ bright layer. The amount of nitrogen in the cap is too small to be detected using EDS.

It should be noted that the high concentration of arsenic in S2 below the cap layer makes it difficult for identifying the composition. The SIMS decay length, measured on the down-slope of the arsenic distribution $(1.8 \mathrm{~nm} /$ decade), is the depth resolution limit for the adopted sputtering conditions. Thus, it is hard to distinguish from the SIMS profiles alone whether there is an actual deposition of arsenic or a proper implantation in the substrate. For 
this reason, STEM and XPS were used to identify the surface layers and measure their compositions.

Figure 2 is a dark field STEM image and an EDS line scan of the capped sample. Two distinct layers are observed - a $\sim 3 \mathrm{~nm}$ amorphous arsenic rich layer which appears bright in the dark field STEM mode and a silicon nitride capping layer. The EDS line scan was measured over a distance of $10 \mathrm{~nm}$, with $0.5 \mathrm{~nm}$ steps and an acquisition time of 15 seconds per step, as shown in the bottom part of Figure 2. The EDS result confirms that the $\sim 3 \mathrm{~nm}$ bright layer is arsenic rich, where the other two main constituents are silicon and oxygen. At the center of the $\sim 3 \mathrm{~nm}$ film, the measured composition is 45 atomic \% As in a silicon and oxygen matrix.

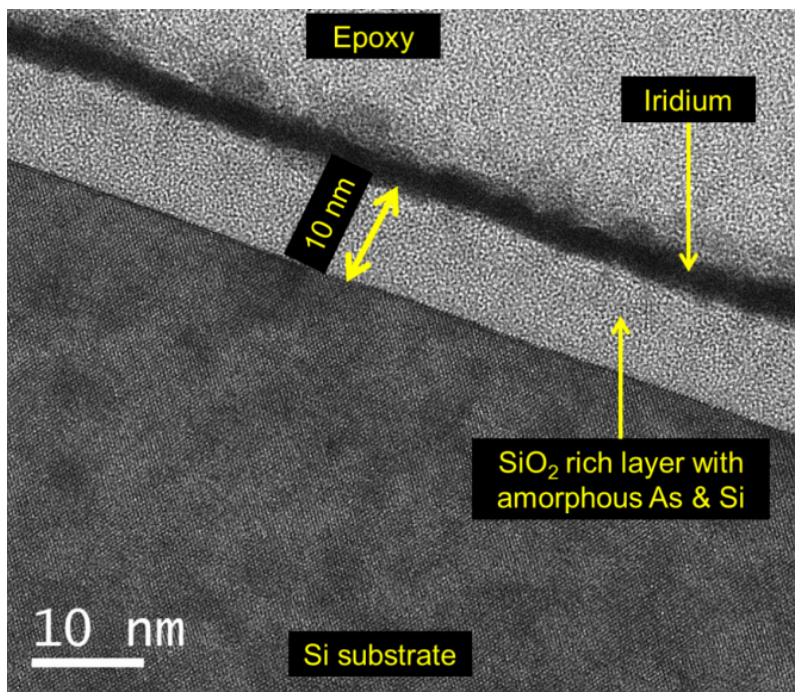

Figure 3. TEM bright field image of S1 (uncapped sample) imaged 7 months after the PIII process. The arsenic enhanced silicon oxide is about $10 \mathrm{~nm}$ thick.

The evolution of the uncapped sample (S1) is rather different, exhibiting a relatively thick native oxide growth. Figure 3 is the TEM cross section, imaged after 200 days of atmospheric exposure. An iridium layer is deposited to distinguish the surface prior to preparing the cross section with a focused ion beam. For this analysis, a region far away from any arsenolite crystal was chosen. In the image, a $10 \mathrm{~nm}$ silicon oxide layer is clearly visible on the silicon substrate, in line with previously observed SIMS and XPS results $[9,10]$. This layer clearly associated with atmospheric exposure since it is not observed in the capped sample, Figure 2.

Further quantitative evaluation of the layer composition was obtained by XPS. To investigate the oxide evolution on the Si surface, measurements on S1 were carried out on 
cleaned samples (micro crystals removed). Figure $4 a$ shows the Si $2 p$ photoelectron spectra of samples S2 and S1, measured after 36 and 129 days of atmospheric exposure. The Si $2 p$ spectrum for sample $\mathrm{S} 2$ shows a main component (the $2 \mathrm{p}_{3 / 2}-2 \mathrm{p}_{1 / 2}$ doublet) at $\sim 100 \mathrm{eV}$ due to $\mathrm{Si}-\mathrm{Si}$ bonds together with a peak at $102 \mathrm{eV}$ due to $\mathrm{Si}-\mathrm{N}$ bond, characteristic of $\mathrm{Si}_{3} \mathrm{~N}_{4}$. A third smaller component at $\sim 103 \mathrm{eV}$ is due to $\mathrm{Si}-\mathrm{O}$ bonds in a non-stoichiometric $\mathrm{SiO}_{\mathrm{x}}$ oxide. The $\mathrm{Si}-\mathrm{N}$ and $\mathrm{Si}-\mathrm{O}$ bonds are due to the ultra-thin cap layer confirming its composition as $\mathrm{SiO}_{x} \mathrm{~N}_{y}$. On the other hand, the S1 spectrum has only two main components, the Si doublet and the peak related to $\mathrm{Si}-\mathrm{O}$ bonds in $\mathrm{SiO}_{2}$ at the appropriate binding energy. This $\mathrm{SiO}_{2}$ peak, readily visible after 36 days in air, drastically dominates the spectrum after 129 days at the expenses of the elemental $\mathrm{Si}$ doublet. The calculation of the $\mathrm{SiO}_{2}$ thickness as described in [13] and approximating the composition to only $\mathrm{Si}$ and $\mathrm{O}$ estimates a $3.7 \mathrm{~nm}$ oxide thickness after 36 days and $6.1 \mathrm{~nm}$ thickness after 129 days, respectively. It should be noted that these values could underestimate the real oxide thickness, since they do not consider the relevant fraction of As atoms present in the growing layer as depicted by SIMS. After the growth of this oxide, the Si $2 p$ doublet fine structure can be resolved, indicating that most of the $\mathrm{Si}$ left below the oxide is of a higher crystallinity compared to that observed after 36 days of air exposure. This is consistent with the oxide growth presented in the TEM of Figure 3.

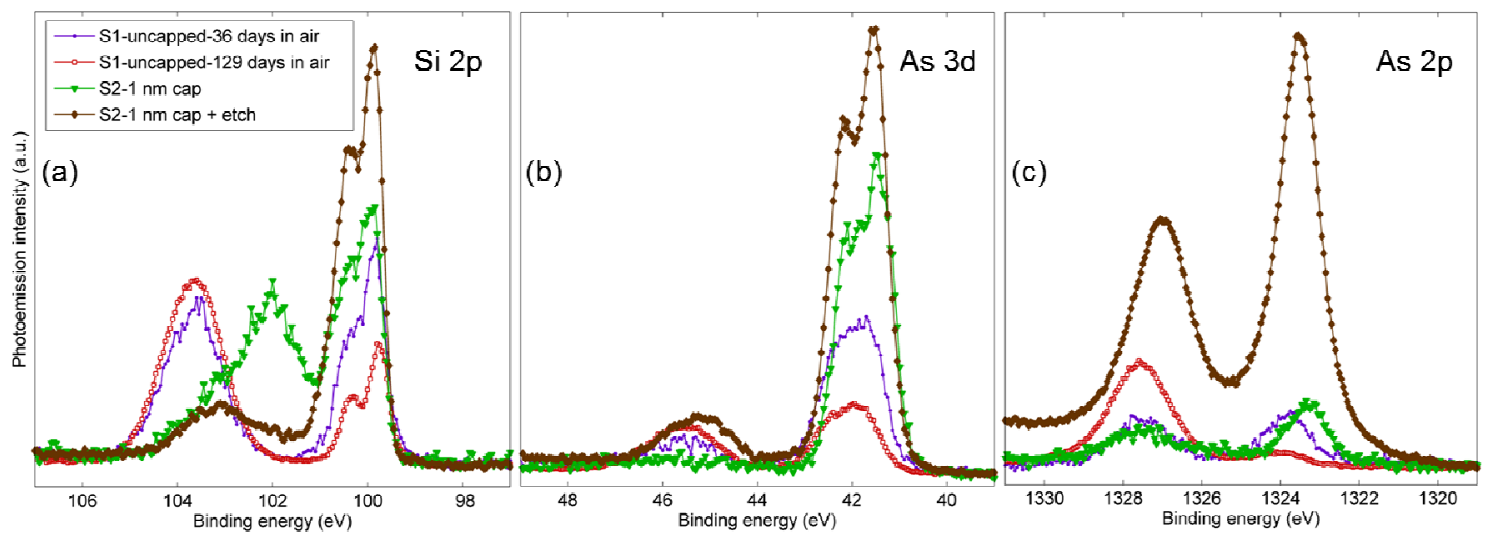

Figure 4. XPS Si $2 p(a)$, As $3 d$ (b) and As $2 p$ core levels for $S 1$ (uncapped) and S2 (capped) samples. S1 was measured after 36 (purple/ circles) and 129 (red/ squares) days of air exposure. S2 was measured before (green/ triangles) and after cap removal by HF wet etch (brown/ diamonds).

Figure $4 b$ depicts the recorded As $3 d$ spectra. It is evident that the cap layer in S2 preserved most of the arsenic from oxidation since only the elemental arsenic component at $41.5 \mathrm{eV}$ can be detected. On the other hand, the uncapped sample shows a peak at $45.5 \mathrm{eV}$ characteristic of As-O bonds, growing with atmosphere exposure time. This confirms that not only silicon oxide grows but also the associated arsenic oxide. The calculated sampling depths for arsenic $3 \mathrm{~d}$ photoelectrons are $9.8 \mathrm{~nm}$ in $\mathrm{Si}, 11.7 \mathrm{~nm}$ in $\mathrm{SiO}_{2}$ and $8.3 \mathrm{~nm}$ in pure 
metallic arsenic. Therefore, the As $3 d$ spectra essentially reports the entire As distribution for all samples, as expected from the SIMS profiles of Figure 1. Aluminum Ka radiation allows the collection of As $2 p$ photoelectrons whose sampling depth is much shallower: $2.1 \mathrm{~nm}$ in $\mathrm{Si}, 2.7 \mathrm{~nm}$ in $\mathrm{SiO}_{2}, 2.3 \mathrm{~nm}$ in $\mathrm{Si}_{3} \mathrm{~N}_{4}$ and $1.9 \mathrm{~nm}$ in metallic arsenic, respectively. The arsenic $2 p$ spectra in Figure $4 c$ therefore provide useful information about the shallower depths. Figure 4c shows two components for all samples: elemental arsenic at $1323 \mathrm{eV}$ and oxidized arsenic at $1327.5 \mathrm{eV}$, respectively. It is interesting to observe that some arsenic oxidation has occurred for sample S2, confirming the presence of oxygen in the thin layer below the cap, as detected by EDS. The sample S1 shows similar intensities for As-As and As-O components already after 36 days of exposure to atmosphere, with the As-O component becoming the dominant one after 129 days, as expected considering the $6 \mathrm{~nm}$ thick oxide.

In order to probe only the arsenic rich amorphous layer, sample S2 was wet-etched and the corresponding XPS spectra are shown in Figure 4. The Si $2 p$ spectrum in Figure $4 a$ shows a dramatic increase of the signal for the Si $2 p$ elemental doublet and a small component at $103.6 \mathrm{eV}$ due to the formation of $\mathrm{SiO}_{\mathrm{x}}$. The latter may also be due to oxidation occurring sample transfer from the wet-etch solution to the XPS chamber ( 20 minutes before the surface was in ultra-high vacuum). Both As $3 d$ and As $2 p$ intensities are much higher compared to all the other recorded spectra, implying that high As concentrations are now being probed. It is interesting to note that both As $3 d$ and As $2 p$ core levels show relevant components due to As-O bonds, although in As $2 p$ now the 'elemental As' peak is higher than that of 'As-O'.

Table II.

\begin{tabular}{|c|c|c|c|c|c|}
\hline \multirow{2}{*}{ Sample } & \multirow{2}{*}{ Cap } & \multirow{2}{*}{$\begin{array}{c}\mathrm{SiO}_{2} \\
\text { thickness } \\
(\mathrm{nm})\end{array}$} & \multicolumn{3}{|c|}{ 'As3d quantitative' } \\
\hline & & & $\mathrm{O}(\%)$ & Si (\%) & As (\%) \\
\hline$\overline{\mathrm{S} 1^{\mathrm{c}}}$ & - & 3.7 & 43.6 & 42.7 & 13.8 \\
\hline$S 1^{d}$ & & 6.1 & 53.7 & 37.6 & 8.7 \\
\hline S2 & Yes & - & 23.6 & 57.0 & 19.5 \\
\hline S2-etched ${ }^{\mathrm{e}}$ & - & 1.2 & 21.0 & 49.7 & 29.3 \\
\hline
\end{tabular}

In Table II we summarize the relative concentrations of oxygen, arsenic and silicon in sample S1 and S2 in different conditions. It is important to mention that we chose the arsenic $3 \mathrm{~d}$ core level for the quantification, so that the electron escape depth is similar for the three elements considered. These results show that $\mathrm{S} 2$ contains half the oxygen compared to $\mathrm{S} 1$, 
confirming the effectiveness of the $\mathrm{SiN}_{\mathrm{x}}$ cap as a protective barrier. At the same time, a larger As concentration is observed for sample S2 than for S1, i.e. $19.5 \%$ versus $13.8 \%$, confirming that a relevant fraction of $\mathrm{As}$ in $\mathrm{S} 1$ is lost due to arsenolite micro crystal formation. However, the S2 As concentration (19.5\%) is lower than the value of $45 \%$ measured by STEM-EDS. This is possibly due to the averaging of the As integral over the full XPS sampled depth. The fraction of As drastically increases in S2 after the HF etch, reaching the value of $29.3 \%$, as expected from a complete removal of the cap.

\subsection{LAYER STRUCTURE AND PHASE}

Raman spectroscopy analysis was carried out on samples S1 and S2. The spectra presented in Figure 5 were taken after 14 and 42 days of atmospheric exposure. The $\mathrm{Si}$ reference spectrum shows only the active second order transverse acoustic (Si 2TA) mode peak at $303.1 \mathrm{~cm}^{-1}$. In addition to this peak, the spectrum from sample S2 shows a broad peak around $233 \mathrm{~cm}^{-1}$ characteristic of the amorphous arsenic phase and a band around $185 \mathrm{~cm}^{-1}$, possibly from an As-O-As bending vibration. Both the 303.1 and $185 \mathrm{~cm}^{-1}$ features are emanating from the arsenic rich layer observed in the STEM cross section [17]. The amorphous arsenic peak is also observed in the $S 1$ spectrum after 14 days, but not the AsO-As one. This may be due to a lower concentration of As in the growing native oxide of S1 compared to S2, and thus the As-O-As signal would be below the detection limit. Spectra collected after 14 and 42 days of atmospheric exposure are nearly identical on S2 sample indicating that the integrity of the $\mathrm{SiN}_{\mathrm{x}}$ cap is maintained thus preserving the arsenic dose. On the other hand, the $\mathrm{S} 1$ clearly shows a decrease in amorphous arsenic concentration, with a spectrum quite similar to the $\mathrm{Si}$ reference, just after 42 days. 


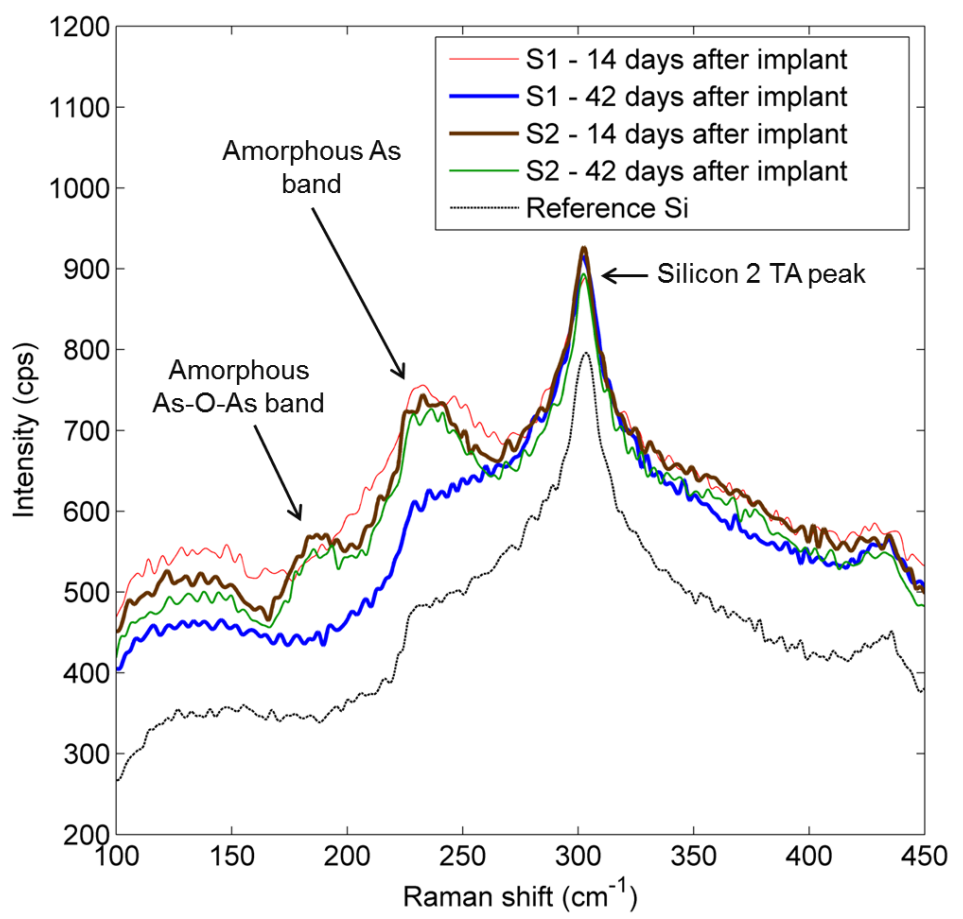

Figure 5. Raman spectra of S1 (un-capped), S2 (capped) samples are compared 14 and 42 days after PIII. The capping layer prevents the loss of arsenic, which can be clearly seen by comparing the amorphous As band for S1 and S2.

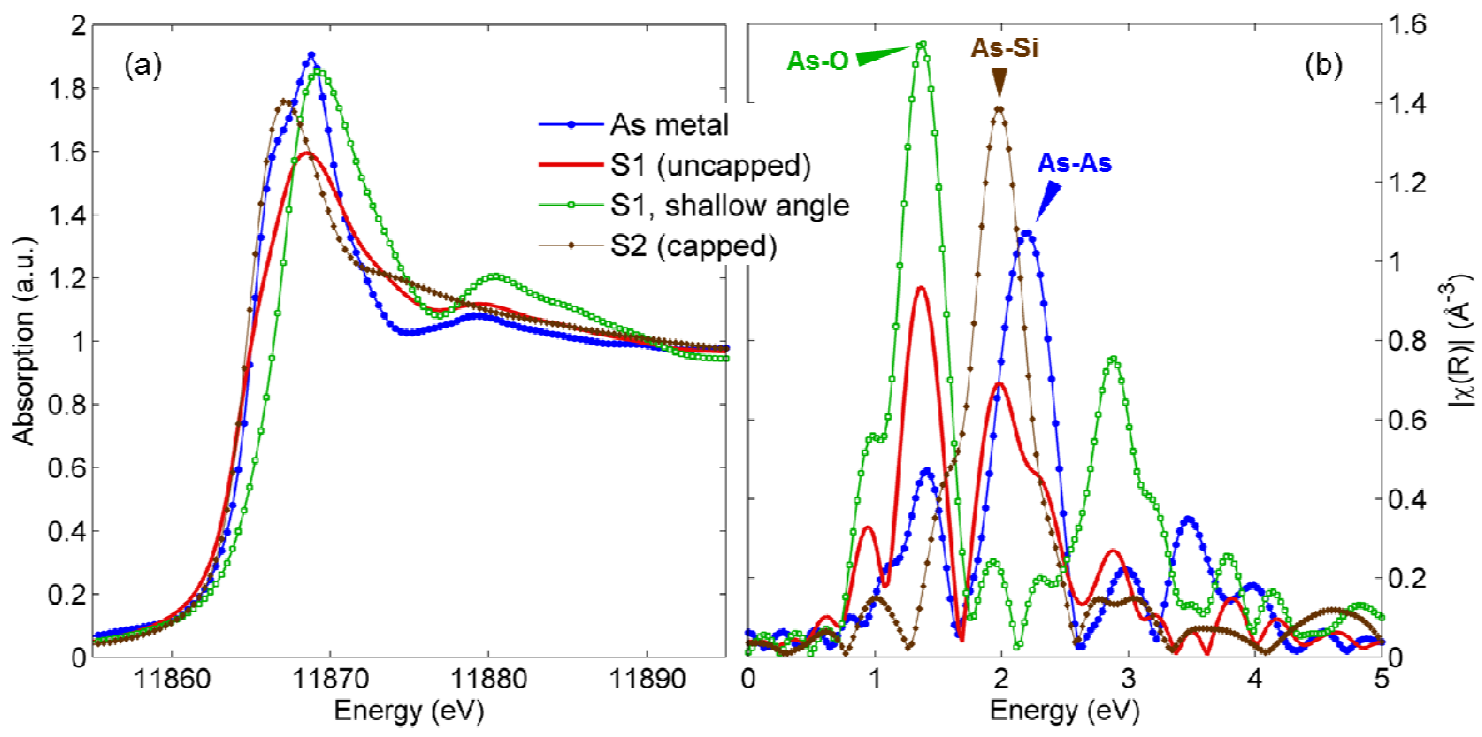

Figure 6. X-ray Absorption Near Edge Structure (a) and the Fourier transform (FT) of the Extended Xray Absorption Fine Structure (b) of sample S1 (uncapped), S2 (capped) and a reference of metallic arsenic. S1 sample was measured at two angles: above and below ('shallow') the critical angle of total external reflection of $\mathrm{x}$-rays, in order to probe different depths of the As distribution. All other measurements were carried out above critical angle to probe the whole implant. 


\subsection{LAYER EVOLUTION: KINETICS OF $\mathrm{As}_{2} \mathrm{O}_{3}$ MICRO-CRYSTAL FORMATION}

The $\mathrm{S} 1$ surface is covered with a distribution of arsenolite micro crystals as reported elsewhere $[9,10]$. Raman spectroscopy was used to monitor the nucleation and growth of the micro crystals on the S1 surface after atmospheric exposure. The Raman spectrum clearly identifies the micro crystal phase as arsenolite (confirming EXAFS data), which is the cubic crystalline phase of arsenic tri-oxide [21] as shown in Figure 7. The arsenolite phase has the following Raman active modes - two $A_{1 g}$, two $E_{g}$, four $T_{2 g}$ and few other combination modes. The $T_{2 g}$ mode observed at $84 \mathrm{~cm}^{-1}$ could be due to a translational mode, since they normally have low Raman shift [21]. All the Raman modes have been identified and listed in Table III. This is in agreement with the results from Gilliam [21] and Takagaki [22]. Silicon has only one first order Raman active mode at $520 \mathrm{~cm}^{-1}$ at the Brillouin zone center from the triply degenerate optical phonons.

The nucleation and growth behavior of every crystal is slightly different from the rest. This may be due to the difference in amorphous arsenic concentration around the seed crystal and the local relative humidity. It also depends on the initial crystal structure; some facets grow faster than others. The changes in the Raman spectra for a typical micro crystal are depicted in Figure 8. The evolution of the arsenolite peak at $368.8 \mathrm{~cm}^{-1}$ with respect to atmospheric exposure is presented in Figure 8a. At day 2, the crystal is still in a rapid growth stage, consuming most of the amorphous arsenic, which can be observed in Figure $8 \mathrm{~b}$. As the crystal continues to grow, consuming the amorphous arsenic in the surrounding regions, the intensity of the arsenolite main peak gets higher. It takes about 10 days for completing the arsenolite crystal growth. 


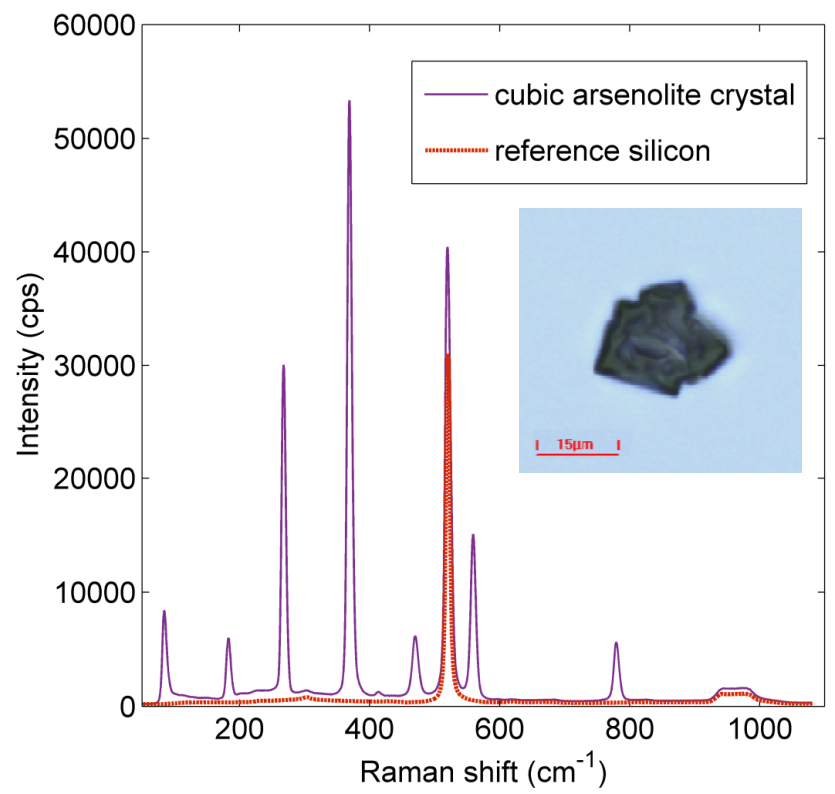

Figure 7. Raman spectrum for a micro crystal (insert) on the surface of $S 1$, measured 13 days after PIII implantation. The spectrum identifies the micro-crystal as arsenolite.

Table III. Peak identification for the Raman spectrum of arsenolite. $(x)+(y)$ refers to a combination of the corresponding modes from peaks $\mathrm{x}$ and $\mathrm{y}$.

\begin{tabular}{|c|c|c|c|}
\hline Peak & $\begin{array}{l}\text { Phonon } \\
\text { mode }\end{array}$ & $\begin{array}{l}\text { Raman } \\
\text { shift }\left(\mathrm{cm}^{-1}\right)\end{array}$ & $\begin{array}{l}\text { Relative } \\
\text { intensity (\%) }\end{array}$ \\
\hline 1 & $\begin{array}{l}\mathrm{T}_{2 \mathrm{~g}}, \\
\text { trans. } \\
\text { mode }\end{array}$ & 84 & 15.75 \\
\hline 2 & $E_{g}$ & 182.8 & 11.23 \\
\hline 3 & $T_{2 g}$ & 267.5 & 56.3 \\
\hline 4 & $A_{1 g}$ & 368.8 & 100 \\
\hline 5 & $\mathrm{~T}_{2 \mathrm{~g}}$ & 413.6 & 2.39 \\
\hline 6 & $\mathrm{~T}_{2 g}$ & 470 & 11.56 \\
\hline 7 & $A_{1 g}$ & 559.1 & 28.3 \\
\hline 8 & (2) $+(5)$ & 596.4 & 1.19 \\
\hline 9 & $(3)+(5)$ & 681.2 & 1.09 \\
\hline 10 & $\mathrm{~T}_{2 \mathrm{~g}}$ & 779.3 & 10.51 \\
\hline 11 & (5) $+(5)$ & 825.2 & 1.11 \\
\hline
\end{tabular}



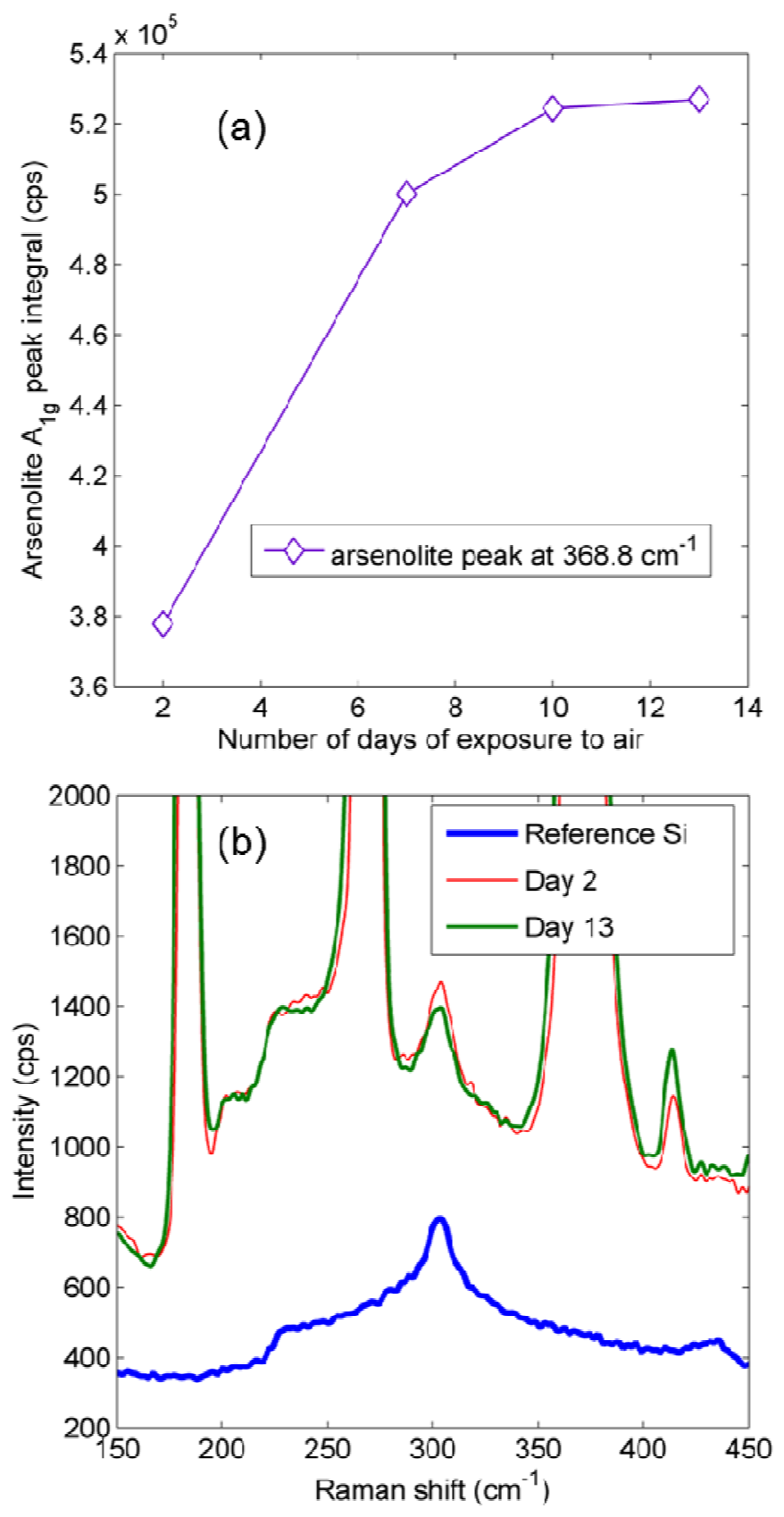

Figure 8. The evolution of Raman spectra during the crystal growth on S1 sample surface. The Raman peak intensity for arsenolite increases during the growth, as shown in (a). Due to a rapid crystallization, amorphous arsenic is consumed early, as shown in (b).

Finally, optical microscopy analysis was carried out to monitor the growth of micro crystals on the $\mathrm{S} 1$ surface over a $2.7 \mathrm{~mm}^{2}$ area, as reported in Figure 9a, which depicts the evolution of the areal density. No relevant micro crystals were observed right after PIII confirming that they grow outside the implantation chamber and not during $\mathrm{AsH}_{3}{ }^{+}$irradiation. However, given the limited resolution of the microscope, it is not possible to exclude an initial 
nucleation at the first atmospheric exposure. Two days after PIII, 4 crystals clearly appeared [9] and the areal density of crystals increased rather quickly in the first week, which progressively saturated after 10 days. The growth of a micro crystal was monitored by the optical profilometer over a period of 11 days, which is reported in Figure 9b. The crystal grew to $4.5 \mu \mathrm{m}$ in height and $15.9 \mu \mathrm{m}$ in diameter. This particular crystal had a high initial growth rate, consuming most of the amorphous arsenic around the seed crystal. After day 7 , the growth rate slowed down, and it reached saturation after day 13.
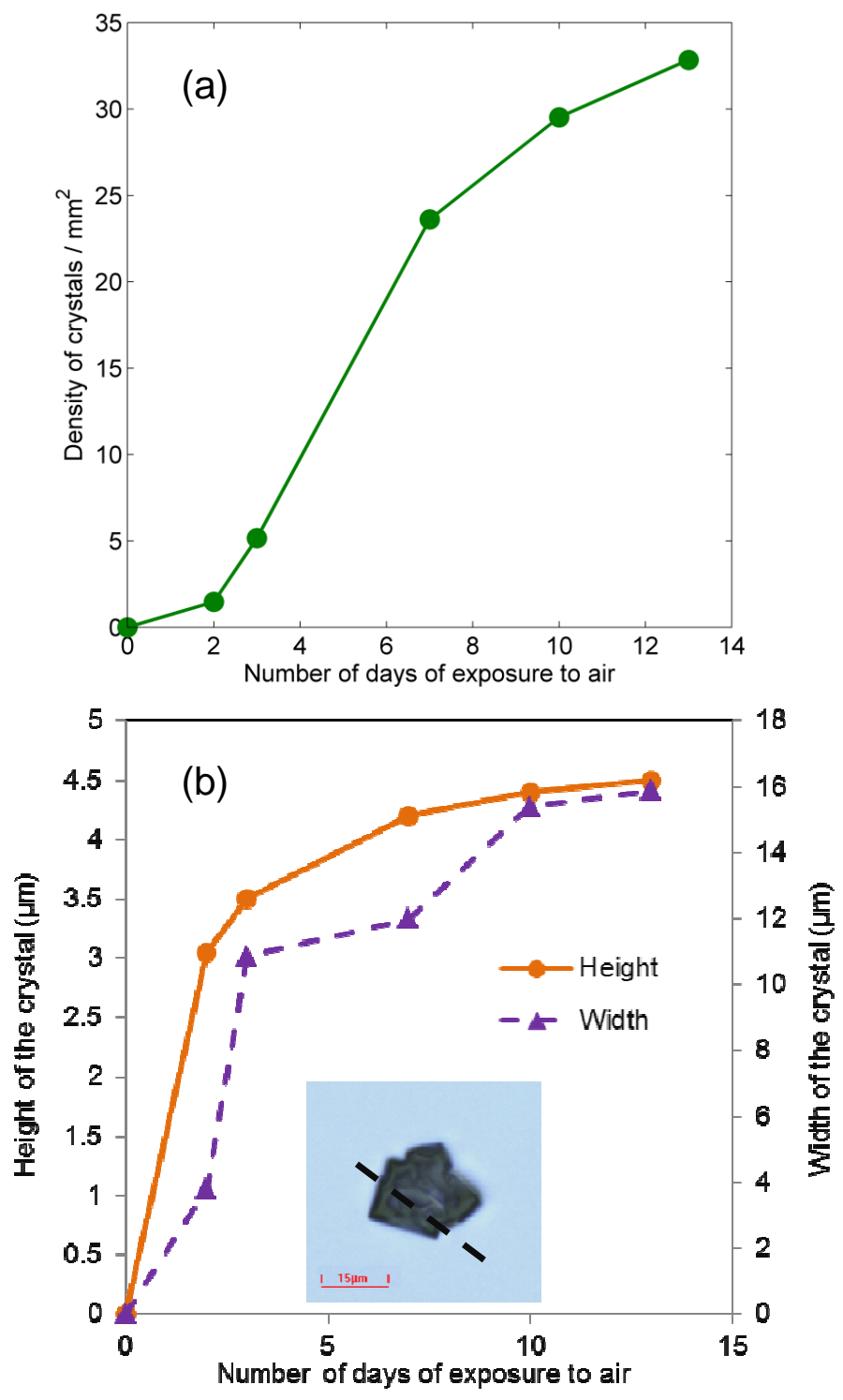

Figure 9 (a) The areal density of micro crystals measured by the optical profilometer on the surface of sample S1. (b) An optical image of an arsenolite crystal observed on S1 surface. The height and width of the crystal (measured along the dotted line) were monitored over a period of 11 days. 


\section{DISCUSSION}

The high fluence $\mathrm{AsH}_{3}{ }^{+} \mathrm{PIII}$ irradiation clearly resulted in the formation of an ultra-thin $\sim 3 \mathrm{~nm}$ amorphous layer as depicted in Figure 2 for sample S2. The layer composition was estimated from STEM-EDS and XPS to be 45 atomic \% arsenic, in a silicon and oxygen matrix. The relevant $\mathrm{Si}$ concentration in this layer means that the observed layer is not simply deposited but the result of ion mixing due to the $\mathrm{AsH}_{3}{ }^{+}$ion implantation. High fluence irradiation results in a high arsenic concentration at surface increasing the average stopping power of the surface for impinging $\mathrm{AsH}_{3}{ }^{+}$ions thus progressively enriching the surface with arsenic. The presence of this arsenic rich layer has two main consequences. (1) The highly reactive surface will consequently form $\mathrm{As}_{2} \mathrm{O}_{3}$ micro crystals and a thick native oxide when exposed to the atmosphere. (2) Only a fraction of the dopant is effectively implanted in the $\mathrm{Si}$ substrate, leading to a self-limiting energy-dependent maximum fluence. The surface $\mathrm{Si}$ is amorphized given the high fluence of energetic arsenic ions, since, for instance, at energies as low as $2.5 \mathrm{keV}$ amorphization can occur with fluences higher than $9 \times 10^{13} \mathrm{ion} / \mathrm{cm}^{-2}(\sim 4 \mathrm{~nm}$ thick amorphous layer) [23]. However, the Si $2 p$ spectra of S1 after 129 days show a typical 'doublet' of a crystalline Si. Therefore, it can be speculated that the high surface reactivity produced a thick native oxide consuming the remaining previously amorphized Si layer.

It has been proposed that the mechanism behind the formation of arsenolite on high fluence $\mathrm{AsH}_{3}{ }^{+}$PIII Si surfaces involved the presence of an ultra-thin film rich in arsenic, as deduced from SIMS profiles [7] and other GaAs based systems [22]. The formation and evolution of arsenolite micro crystals seems to follow the typical crystal growth kinetics where at the beginning there is a good supply of reacting agents, at least one of which is progressively consumed. This is clearly revealed by the evolution of Raman spectra of sample S1 (uncapped) where the 'amorphous-As' peak observed at $233 \mathrm{~cm}^{-1}$ is progressively decreasing with atmospheric exposure whereas the peaks related to $A s_{2} \mathrm{O}_{3}$ clearly grow. Since oxygen can be considered in constant supply in air, one can assume that the kinetics is thus driven by the supply of arsenic available on the irradiated surface. The size of crystals could then be influenced by the initial nucleation seed, the presence of amorphous arsenic and surface migration from the surrounding region, and the relative humidity in the atmosphere.

It is interesting to note that there is a small time-shift between the growth saturation of the crystal monitored in Figure $9 \mathrm{~b}$ and the saturation of the areal density of micro crystals reported in Figure 9a. The latter was not yet reached after 12 days of air exposure, whereas the crystal growth saturated after 6-10 days. This suggests that a limiting factor for the 
crystal growth may be the surface diffusion of arsenic atoms at room temperature and not only the presence of the arsenic rich layer.

Since this work confirmed that the $\operatorname{SiN}_{x}$ cap was able to preserve the arsenic rich layer induced by PIII, a HF wet-etch was performed to expose the original implanted surface to atmosphere and possibly monitor arsenolite nucleation. However, it appears that the chemical etch was not completely selective with respect to the arsenic implanted surface, leaving only $3.6 \times 10^{15} \mathrm{at} / \mathrm{cm}^{2}$ of the original $5.7 \times 10^{15}$ As fluence, according to INAA measurement (Table I). The sample surface was then monitored by SEM and optical microscopy over a period of 1 month. No crystal formation was observed during this time, suggesting that the reduced amount of arsenic left after etching was not sufficient to allow for the arsenolite nucleation. On the other hand, it is clear that the $\mathrm{As}_{2} \mathrm{O}_{3}$ formation and the subsequent dopant loss are triggered by the arsenic out-diffusion to the surface, since oxygen is in constant supply in the atmosphere.

The other striking observation linked to the 'arsenic rich' layer is the relatively thick and unstable native oxide observed on PIII samples like $S 1[9,10]$. This is a matter of a future study but it is clear that the high level of arsenic concentration acts as a catalyst for $\mathrm{Si}$ oxidation, an effect already observed for lower arsenic concentration in Si but only at higher temperatures [24-27].

\section{CONCLUSION}

High-fluence $\left(>1 \times 10^{15} \mathrm{at} / \mathrm{cm}^{2}\right)$ low energy $\mathrm{AsH}_{3}{ }^{+}$PIII of (100) Si surface resulted in the formation of an ultra-thin ( $3 \mathrm{~nm})$ amorphous layer, whose composition is $45 \%$ arsenic in a silicon and oxygen matrix. This layer was revealed using samples prepared by depositing insitu a nanometer thick $\mathrm{SiN}_{\mathrm{x}}$ cap layer right after PIII and before exposing the irradiated surface to atmosphere. The layer is thought to be the result of a combined effect of ion mixing and arsenic surface enrichment, triggered by the extremely high arsenic concentration achieved at the Si surface during the first phases of $\mathrm{AsH}_{3}{ }^{+}$irradiation. This high arsenic concentration results in progressively lower penetration of impinging ions, leading to a prevalence of arsenic over the implanted profile.

The arsenic rich layer is highly reactive when exposed to atmosphere. In particular, arsenic reacts with atmospheric oxygen and moisture forming arsenolite micro crystals, whereas $\mathrm{Si}$ is quickly oxidized thanks to a catalytic role of arsenic atoms resulting in thick (>7 nm) and unstable native oxides. The micro crystal formation consumes half of the originally implanted arsenic. This arsenic will be lost since it is not possible to drive in the 
dopant atoms, due to the easy sublimation of $\mathrm{As}_{2} \mathrm{O}_{3}$, at temperatures as low as $200{ }^{\circ} \mathrm{C}$. During the formation of the thick native oxide another relevant fraction of the dopant is locked into the growing oxide and thus rendered electrically inactive. However, if a high level of dopant concentration is required for technological reasons; two solutions can be suggested based on this study. The thermal treatment should be performed as soon as PIII is completed, before any $\mathrm{As}_{2} \mathrm{O}_{3}$ crystal formation and enhanced $\mathrm{Si}$ oxidation that are triggered by prolonged atmosphere exposure. Alternatively, the deposition of an ultra-thin cap, such as $\mathrm{SiN}_{\mathrm{x}}$ inhibits the reactivity of the surface with atmosphere and successive thermal treatments may be able to diffuse arsenic atoms into Si. A third option was recently reported by Meirer et al. [9], where $\mathrm{AsH}_{3}{ }^{+} \mathrm{PIII}$ had been carried out in an oxygen controlled plasma during implantation. This resulted in a lower implanted arsenic fluence, i.e. $\sim 2.5 \times 10^{15}$ compared to $\sim 6 \times 10^{15}$ for a normal oxygen conditioned PIII in that study as measured by INAA. However, the result was a more stable surface, without the appearance of $\mathrm{As}_{2} \mathrm{O}_{3}$ micro-crystals and a stable, thinner native oxide $(\sim 4 \mathrm{~nm})$.

\section{Acknowledgement}

We thank Matthew Castle and Martin Hilkene for their assistance with performing the arsenic PIII. The activity of FM was supported by the Autonomous Province of Trento under the framework of the DART project, Marie Curie - COFUND Program. X-Ray absorption measurements were carried out at the Stanford Synchrotron Radiation Lightsource (SSRL), a national user facility operated by Stanford University on behalf of the U.S. Department of Energy, Office of Basic Energy Sciences.

\section{References}

[1] Y. Sasaki, K. Okashita, B. Mizuo, M. Kubota, M. Ogura, and O. Nishijima, Journal of Applied Physics 111, 013712 (2012).

[2] A. K. Kambham, A. Kumar, A. Florakis, and W. Vandervorst, Nanotechnology 24, 275705 (2013).

[3] D. Gupta, International Journal of Advancements in Technology 2, 471 (2011).

[4] E.C. Jones, W. En, S. Ogawa, D.B Fraser and N.W. Cheung, Journal of Vacuum Science and Technology B, 12, 956 (1994).

[5] S. Qin, K. Zhuang, Y. J. Hu, A. McTeer, and S. Lu, IEEE Transactions on Plasma Science 37, 2082 (2009).

[6] S. Felch, Z. Fang, B.-W. Koo, R. . Liebert, S. . Walther, and D. Hacker, Surface and Coatings Technology 156, 229 (2002). 
[7] F. Meirer, E. Demenev, D. Giubertoni, S. Gennaro, L. Vanzetti, G. Pepponi, M. Bersani, M. A. Sahiner, G. Steinhauser, M. A. Foad, J. C. Woicik, A. Mehta, and P. Pianetta, in AIP Conference Proceedings (2012), pp. 183-188.

[8] M. Kubota, S. Hayashi, M. Ogura, Y. Sasaki, S. Okada, and K. Yamabe, Japanese Journal of Applied Physics 52, 021301 (2013).

[9] F. Meirer, D. Giubertoni, E. Demenev, L. Vanzetti, S. Gennaro, M. Fedrizzi, G. Pepponi, A. Mehta, P. Pianetta, G. Steinhauser, V. Vishwanath, M. Foad, and M. Bersani, Applied Physics Letters 101, 232107 (2012).

[10] F. Meirer, E. Demenev, D. Giubertoni, L. Vanzetti, S. Gennaro, M. Fedrizzi, G. Pepponi, G. Steinhauser, V. Vishwanath, M. Foad, and M. Bersani, Physica Status Solidi (C) 11, 28 (2014).

[11] E. Demenev, D. Giubertoni, J. van den Berg, M. Reading, and M. Bersani, Nuclear Instruments and Methods in Physics Research Section B: Beam Interactions with Materials and Atoms 273, 192 (2012).

[12] W. Adlassnig, G. Steinhauser, M. Peroutka, A. Musilek, J.H. Sterba, I.K. Lichtscheidl, M. Bichler, Appl. Radiat. Isot. 67, 2117 (2009).

[13] M. P. Seah and S. J. Spencer, Surface and Interface Analysis 33, 640 (2002).

[14] F. Benzi, I. Davoli, M. Rovezzi, and F. d'Acapito, The Review of Scientific Instruments 79, 103902 (2008).

[15] J. Kawai, S. Hayakawa, Y. Kitajima, and Y. Gohshi, Spectrochimica Acta Part B: Atomic Spectroscopy 54, 215 (1999).

[16] D. Giubertoni, G. Pepponi, S. Gennaro, M. Bersani, M. A. Sahiner, S. P. Kelty, R. Doherty, M. a. Foad, M. Kah, K. J. Kirkby, J. C. Woicik, and P. Pianetta, Journal of Applied Physics 104, 103716 (2008).

[17] G. P. Schwartz, J. E. Griffiths, D. DiStefano, G. J. Gualtieri, and B. Schwartz, Applied Physics Letters 34, 742 (1979).

[18] P. Ballirano and A. Maras, Zeitschrift Für Kristallographie-New Crystal Structures 217, 177 (2002).

[19] G. Cargill, Journal of Non-Crystalline Solids 150, 347 (1992).

[20] A. Foster and G. B. Jr, American Mineralogist 83, 553 (1998).

[21] S. J. Gilliam, C. N. Merrow, S. J. Kirkby, J. O. Jensen, D. Zeroka, and A. Banerjee, Journal of Solid State Chemistry 173, 54 (2003).

[22] Y. Takagaki, E. Wiebicke, M. Ramsteiner, L. Däweritz, and K. H. Ploog, Applied Physics A: Materials Science \& Processing 76, 837 (2003).

[23] M. Werner, J. Vandenberg, D. Armour, W. Vandervorst, E. Collart, R. Goldberg, P. Bailey, and T. Noakes, Nuclear Instruments and Methods in Physics Research Section B: Beam Interactions with Materials and Atoms 216, 67 (2004).

[24] S. S. Choi, M. Z. Numan, W. K. Chu, J. K. Srivastava, and E. a. Irene, Applied Physics Letters 50, 688 (1987). 
[25] S. S. Choi, M. Z. Numan, W. K. Chu, and E. a. Irene, Applied Physics Letters 51, 1001 (1987).

[26] S. Ohkawa and Y. Nakajima, Journal of Electrochemical Society 125, 1997 (1997).

[27] E. Biermann, H. H. Berger, P. Linke, and B. Muller, Journal of the 143, 1434 (1996).

[28] G. Steinhauser, J. Clean. Prod. 8, 833 (2008). 